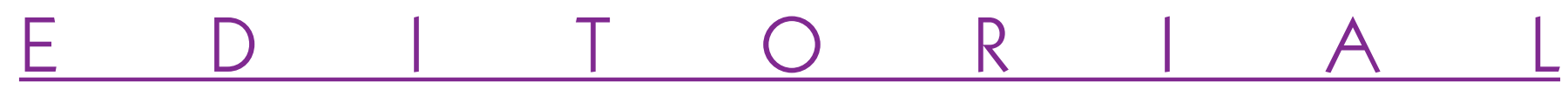

\title{
What has happened to the old time fundamentals?
}

\author{
Jo all those who have been perplexed by cases of malocclusion of the teeth \\ and have not become discouraged thereby...
}

Edward H. ANGLE, 1898.

Once upon a time in the good old days charming young girls who wanted to get their teeth straightened willingly agreed to wear a head cap for days and months on end and to have a metal arch wire with two gross elastics attached to it installed their mouths. The goal of this unsightly burden, orthodontists explained to them, was to move their upper first molars back far enough to make room in their dental arches for all the other teeth to be evenly aligned.

Thirty years later, we seem to have decided that it is impossible, or inadvisable, to distalize the maxillary molars past the pterygoid processes with the aid of "EOF" or of that other device called "The Pendulum".

Why did it take us so long to discover the inefficacy of this procedure?

The Dutch and the Japanese have, notably, for years followed the Begg technique which postulates the immediate removing not moving of two upper molars.

As the years go by we never see a patient wearing an EOF appliance. What's happened?

What has happened is that a new generation of adolescents is no longer willing to put up with those old fashioned techniques. Adolescence is a period when the body changes, when the dentition changes, and when boys and girls begin to wonder about their futures. They dor t want to follow slavishly in their parents' footsteps and they certainly dont want to be "controlled" by adults or even by their peers. This new generation has discovered sexuality much earlier than their elders did and they are easily tempted by the siren call of the consumption society.

Realizing all this, we have, a little late, replaced the uncool head gear with intraoral elastics which have had only a limited success, owing to the sad reality that they only work when patients wear them.

So orthodontists have adjusted their methods, finding alternative sources of anchorage respecting as much as possible their patients' strong desire to keep their braces invisible to public 
scrutiny. Even if this goal is too often illusory, orthodontists do their best to camouflage their mechanical interventions. They have found more inconspicuous ways to support tooth movements, mini-screws, which, alas, are often unstable. But it is by means of these more or less hidden implants that nowadays many orthodontists establish the force needed to move teeth and obtain the desired result.

Meanwhile, from across the border in Germany the rods and tubes of the Herbst appliance that were re-popularized by Pancherz have begun to find wide acceptance in France. These appliances have a reciprocal action that, like the anchorage based on min-screws, does not depend on the patient's cooperation. In the final analysis, a reasoned score card would generally show obtaining good results with mechanically sound techniques depends not on the motivation of the young patients but far more on the motivation of the orthodontists and how well they construct their fixed appliances.

As for adult orthodontics and, perhaps, later for teen-agers, the German approach with individualized attachments and arch wires, is introducing a remarkable new technological era in orthodontics: it is probable that, in the next few years, the patient's oral landscape will be reproduced by a scanner that will make it possible for brackets and arch wires to be made to measure in accordance with the prescribed orthodontic protocol defined to respond to precise functional and esthetic requirements.

As for inter-arch mechanics...

Pierre PLANCHÉ 